\title{
UNIVERSITY OF TEXAS AT AUSTIN RADIOCARBON DATES XIV
}

\author{
S VALASTRO, JR, E MOTT DAVIS, ALEJANDRA G VARELA, \\ and CAROLYN EKLAND-OLSON \\ Radiocarbon Laboratory, Balcones Research Center, \\ The University of Texas at Austin
}

This list reports certain ${ }^{14} \mathrm{C}$ measurements completed by December 1979; other projects completed by this time will be reported later. Age calculations are based on ${ }^{14} \mathrm{C}$ half-life of $5568 \mathrm{yr}$ and modern standard of $95 \%$ NBS oxalic acid, supplemented by tree rings of pre-industrial wood from a $\log$ cut in the 1850 's (Tx-540; R, 1970, v 12, p 249). Deviations reported are based on counting statistics of sample, background and modern, and are $\pm 1 \sigma$, except that when sample count approaches either modern or background, $2 \sigma$ limits are reported. Unless noted, ${ }^{12} \mathrm{C} /{ }^{13} \mathrm{C}$ measurements were not made and results are not corrected for ${ }^{13} \mathrm{C}$ fractionation (assumed ratio $=-25 \%$ WRT PDB). Our laboratory uses liquid scintillation counting of benzene, with $\mathrm{Li}_{2} \mathrm{C}_{2}$ and vanadium-activated catalyst in preparation; chemical yields range between $95 \%$ and $99 \%$. Three counters are employed; a Packard Tri-Carb Model 3002 and 2 Beckman LS230 spectrometers obtained through a grant from the National Science Foundation.

We acknowledge with gratitude the administrative support and assistance of W G Reeder and Saralind Mings of the Texas Memorial Museum.

\section{OCEANOGRAPHIC SAMPLES}

\section{Bahamas}

\section{Great Bahama Bank series, Bahamas}

Samples of carbonate sediment from slope N of Great Bahama Bank, Bahama Is. Coll to determine origin and rate of deposition of breccia deposits and rates of coral growth. Coll 1978 by A C Neumann and subm by M Boardman, Marine Sci Program, Univ North Carolina, Chapel Hill.

Tx-2997. E-29262/40cm

$4010 \pm 60$ $30 \mathrm{~km} \mathrm{~S}$ of Freeport $\left(26^{\circ} 19^{\prime} \mathrm{N}, 78^{\circ} 33^{\prime} \mathrm{W}\right), 39$ to $41 \mathrm{~cm}$ in core.

\section{Tx-2998. E-29262/80cm}

Same location as $\mathrm{Tx}-2997,79$ to $81 \mathrm{~cm}$ in core.

Tx-2999. E-29262/100cm

Same location as $\mathrm{Tx}-2997,99$ to $101 \mathrm{~cm}$ in core.

Tx-3000. E-29262/20cm

Same location as $\mathbf{T x}-2997,18.5$ to $21.5 \mathrm{~cm}$ from surface. 
Tx-3001. E-29283/7 cm

$15 \mathrm{~km} \mathrm{~S}$ of McLean's Town $\left(26^{\circ} 27^{\prime} \mathrm{N}, 78^{\circ} 05^{\prime} \mathrm{W}\right), 6$ to $8 \mathrm{~cm}$ in core.

\section{Tx-3002. E-29283/20cm}

Same location as $\mathrm{T} x-3001,19$ to $21 \mathrm{~cm}$ in core.

Tx-3003. E-29283/40cm

Same location as $\mathrm{Tx}-3001,39$ to $41 \mathrm{~cm}$ in core.

\section{Tx-3004. E-29283/60cm}

Same location as $\mathrm{Tx}-3001,59$ to $61 \mathrm{~cm}$ in core.

\section{Tx-3005. E-29283/88cm}

Same location as $\mathrm{Tx}-3001,88 \mathrm{~cm}$ in core.

Tx-3006. E-34631/47cm

$40 \mathrm{~km} \mathrm{~S}$ of McLean's Town $\left(26^{\circ} 1 \mathrm{1}^{\prime} \mathrm{N}, 78^{\circ} 07^{\prime} \mathrm{W}\right), 45$ to $50 \mathrm{~cm}$ in core.

\section{Tx-3007. E-34631/7cm}

Same location as $\mathrm{Tx}-3006,5$ to $10 \mathrm{~cm}$ in core.

$$
\begin{array}{r}
\mathbf{3 8 6 0} \pm \mathbf{6 0} \\
\delta^{13} C=+3.5 \% \text { o }
\end{array}
$$

$$
\begin{array}{r}
6550 \pm 90 \\
\delta^{13} C=+3.2 \% o
\end{array}
$$

$$
12,260 \pm 160
$$$$
\delta^{13} \mathrm{C}=+1.6 \% \text { o }
$$

$$
\begin{array}{r}
\mathbf{3 0 , 6 0 0} \pm \mathbf{2 4 0 0} \\
\delta^{13} C=+2.1 \% \text { o }
\end{array}
$$

$$
\begin{array}{r}
\mathbf{3 8 7 0} \pm \mathbf{6 0} \\
\delta^{13} C=+3.6 \% \text { o }
\end{array}
$$

$1790 \pm 60$

$\delta^{13} C=+3.6 \%$

General Comment on Tx-2997 to Tx-3007 (MB): data have been coupled with mineralogic, minor element, and stable isotope data with following results: 1) Holocene deposits have higher aragonite and strontium content than late Pleistocene, calcite-rich sediments; 2) Holocene deposition rates are approx 5 times greater than late Pleistocene rates; 3) major change in mineralogy and minor element concentrations occurs at 5000 to 7000 вP; 4a) isotope data from Holocene sediments are distinctly clumped $\left(\delta^{18} \mathrm{O} \cong-0.5\right.$ to $0.0, \delta^{13} \mathrm{C} \cong+3$ to +4$)$, and this range is indistinguishable from shallow carbonate sediments; $4 \mathrm{~b}$ ) isotope data from Pleistocene sediments are $\delta^{18} \mathrm{O} \cong+0.8$ to $+1.6, \delta^{13} \mathrm{C} \cong+1.5$ to +2.8 . These data strongly support hypothesis that when sea level flooded banktops, offbank transport of carbonate sediments began and now dominate deposition of carbonate sediments in deep channels. Roughly $80 \%$ of deep-sea sediments in NW Providence Channel is of banktop origin.

Tx-3008. 764-F17 $\left.10^{\prime} \mathrm{W}\right)$.

Deep water coral (BASE), $4 \mathrm{~km} \mathrm{~W}$ of Memory Rock $\left(26^{\circ} 50^{\prime} \mathrm{N}, 79^{\circ}\right.$

\section{Tx-3009. 764/F17}

Coral (TIPS), $4 \mathrm{~km} \mathrm{~W}$ of
Ulira modern $\delta^{13} \mathrm{C}=+2.1 \%$

General Comment on Tx-3008 and Tx-3009 (MB): dates demonstrate that this deep-sea (ahermatypic) coral grows quite rapidly. Coral, ca $50 \mathrm{~cm}$ high, appears to have grown $50 \mathrm{~cm}$ in $80 \mathrm{yr}$. 
Tx-3027. E-34652-15-SED

$4650 \pm 70$

NW Providence Channel $\left(25^{\circ} 56^{\prime} \mathrm{N}, 78^{\circ} 31^{\prime} \mathrm{W}\right)$, $405 \mathrm{~m}$ water depth, $15 \mathrm{~cm}$ below sediment-water interface.

Tx-3028. E-34652-110-SED

$22,670 \pm 290$

$\delta^{13} C=+3.2 \%$

Same location as Tx-3027, $110 \mathrm{~cm}$ below sediment surface.

\section{Tx-3029. E-34652-88-RX}

$17,210 \pm 310$

C. ment surface.

Tx-3030. E-34652-140-SED

$26,250 \pm 680$

$\delta^{13} \mathrm{C}=+3.2 \%$

Same location as $\mathrm{Tx}-3027,140 \mathrm{~cm}$ below sediment surface.

\section{Tx-3031. E-34652-88-SED}

$21,260 \pm 4.90$

$\delta^{13} C=+3.3 \%$

Same location as $\mathrm{Tx}-3027,88 \mathrm{~cm}$ below sediment surface.

$$
\begin{array}{ccc} 
& & \mathbf{2 0 , 8 8 0} \pm \mathbf{4 9 0} \\
\text { Tx-3032. E-34652-140-RX } & \delta^{13} C=+3.6 \% o
\end{array}
$$

$\mathrm{CaCO}_{3}$ rock fragments, same location as Tx-3027, $140 \mathrm{~cm}$ below sediment surface.

\section{Tx-3033. E-34658-20-SED}

$8380 \pm 110$

$\delta^{13} C=+3.5 \%$

NW Providence Channel $\left(25^{\circ} 54^{\prime} \mathrm{N}, 78^{\circ} 32^{\prime} \mathrm{W}\right)$, 295m core depth, $20 \mathrm{~cm}$ deep in core.

Tx-3034. E-34658-80-RX

$13,880 \pm 150$

$\mathrm{CaCO}_{3}$ rock fragments, same location as $\mathrm{Tx}-3033,80 \mathrm{~cm}$ deep in core.

\section{Tx-3035. E-34664}

$9970 \pm 90$

$\delta^{13} \mathrm{C}=+3.2 \%$

$\mathrm{CaCO}_{3}$ rock, NW Providence Channel (25 $\left.53^{\prime} \mathrm{N}, 78^{\circ} 33^{\prime} \mathrm{W}\right), 200 \mathrm{~m}$ water depth.

General Comment on $T x-3027$ to Tx-3035 (MB): dates demonstrate that sediments and rock fragments of breccia zones are late Pleistocene, not Holocene; so modern chemical conditions are of little direct interest in lithification processes of these rock fragments. Rocks are 4000 to $5000 \mathrm{yr}$ younger than surrounding sediment. Deposition rates of these brecciafilled cores are 8 to $15 \mathrm{~cm} / 1000 \mathrm{yr}$; this suggests mass movement of sediment as deposition process.

\section{Abaco Sound series, Bahamas}

Shell and sediment samples from 3 cores of lagoon sediments in Abaco Sound, NE margin of Little Bahama Bank near Great Abaco I., Bahamas. Coll 1977 by A C Neuman and subm by S Locker, Dept Marine Sci, Univ North Carolina, Chapel Hill. Cm figures are depths in core. 
Tx-3229. C7714-18-30

Anodontia alba shells, ca $3.7 \mathrm{~km} \mathrm{~N}$ of Marsh Harbour $\left(26^{\circ} 35^{\prime} \mathrm{N}\right.$, $\left.77^{\circ} 3.9^{\prime} \mathrm{W}\right), 489$ to $493 \mathrm{~cm}$

Tx-3230. C7714-16-22

$4410 \pm 100$

Chione cancellata shells, ca $4.6 \mathrm{~km} \mathrm{NW}$ of Treasure Cay $\left(26^{\circ} 43.7^{\prime} \mathrm{N}\right.$, $\left.77^{\circ} 19.7^{\prime} \mathrm{W}\right), 415$ to $417 \mathrm{~cm}$.

Tx-3231. C7714-14-19

$8230 \pm 260$

Anodontia alba shells, ca $11 \mathrm{~km} \mathrm{NW}$ of Treasure Cay $\left(26^{\circ} 46.7^{\prime} \mathrm{N}\right.$, $\left.77^{\circ} 22.6^{\prime} \mathrm{W}\right), 424$ to $427 \mathrm{~cm}$.

\section{Tx-3232. C7714-14-19}

$4120 \pm 100$ $247 \mathrm{~cm}$

Chione cancellata shells, same location as Tx-3231 above, 245 to

Tx-3233. C7714-14-19

$6940 \pm 80$

$\mathrm{CaCO}_{3}$ sediment, same location as Tx-3231, above, 420 to $422 \mathrm{~cm}$.

Tx-3234. C7714-14-19

$\mathrm{CaCO}_{3}$ sediment, same location as Tx-3231, above, 376 to $378 \mathrm{~cm}$.

$$
6510 \pm 90
$$

Tx-3235. C7714-18-30

$3800 \pm 100$

$\mathrm{CaCO}_{3}$ sediment, same location as $\mathrm{Tx}-3229$, above, 410 to $413 \mathrm{~cm}$.

Tx-3236. C7714-18-30

$3920 \pm 90$

$\mathrm{CaCO}_{3}$ sediment, same location as $\mathrm{Tx}-3229$, above, 310 to $313 \mathrm{~cm}$.

Tx-3237. C7714-14-19

$1190 \pm 80$ $73 \mathrm{~cm}$.

Codakia orbicularis shell, same location as Tx-3231, above, 69 to

Tx-3238. C7714-18-30

$1640 \pm 50$

$\mathrm{CaCO}_{3}$ sediment, same location as $\mathrm{Tx}-3229$, above, 110 to $113 \mathrm{~cm}$.

Tx-3239. C7714-18-30

$1060 \pm 50$

$\mathrm{CaCO}_{3}$ sediment, same location as $\mathrm{Tx}-3229$, above, 10 to $13 \mathrm{~cm}$.

Tx-3240. C7714-16-22

$2040 \pm 140$

$\mathrm{CaCO}_{3}$ sediment, same location as $\mathrm{Tx}-3230$, above, 200 to $202 \mathrm{~cm}$.

Tx-3241. C7714-16-22

$1110 \pm 70$

$\mathrm{CaCO}_{3}$ sediment, same location as Tx-3230, above, 100 to $102 \mathrm{~cm}$.

Tx-3242. C7714-18-30

$1940 \pm 70$

$\mathrm{CaCO}_{3}$ sediment, same location as Tx-3229, above, 210 to $213 \mathrm{~cm}$.

General Comment (SL): sedimentation rate in core C7714-14-19 is ca $58 \mathrm{~cm} / 1000 \mathrm{yr}$ and in Core C7714-16-22 is $101 \mathrm{~cm} / 1000$ yr. In Core C7714$18-30$ rate is $97 \mathrm{~cm} / 1000 \mathrm{yr}$ with increase during last $1000 \mathrm{yr}$ (as indicated by highest 3 dates, Tx-3238, $-3239,-3242$ ) to $300 \mathrm{~cm} / 1000 \mathrm{yr}$, comparing with $\mathrm{Pb}-210$ data from Core C7714-16-22 indicating maximum rate of $300 \mathrm{~cm} / 1000 \mathrm{yr}$. 


\section{Tx-2561. MGM 1CD1, Nevada}

Wood from E wall MGM GRAND-Reno Pit site, $34 \mathrm{~m}$ depth, Reno, Nevada $\left(39^{\circ} 31^{\prime} 15^{\prime \prime} \mathrm{N}, 119^{\circ} 46^{\prime} 30^{\prime \prime} \mathrm{W}\right)$. NW 1/4, NE 1/4, Sec 18, T 19N, R20E, MDBM. Coll 1976 and subm by J Bell, Nevada Bur Mines \& Geol, Univ Nevada, Reno. Comment (JB): date supports interpretation that outwash deposit is Tahoe (early Wisconsinan) age.

$$
\text { Mexico, Venezuela }
$$

\section{Tlapacoya series, Mexico}

Dark lacustrine mud from Trench Beta, SE side Cerro Tlapacoya, $\mathrm{N}$ of Puebla toll rd ca $28 \mathrm{~km}$ SE of Plaza de Constitucion, D F, Mexico $\left(19^{\circ} 17^{\prime} 30^{\prime \prime} \mathrm{N}, 98^{\circ} 55^{\prime} 00^{\prime \prime} \mathrm{W}\right)$. Coll to establish ages for sediments used in paleomagnetic investigation. Coll 1973 by P W Lambert, U S Geol Survey, and subm by Lambert and Valastro, this lab.

\section{Tx-1784. Tlapacoya, 73L61}

NE side of trench, $23 \mathrm{~m}$ from reference point.

Tx-1913. Tlapacoya, $12 / 15 / 73 / 1$

$$
\mathbf{1 4 , 5 4 0} \pm 90
$$

$\mathrm{N}$ wall of trench, $30 \mathrm{~m}$ from reference point. Date is average; sample split and 2 parts prepared and counted separately: 14,450 $\pm 120,14,630$ \pm 130 .

Tx-1914. Tlapacoya $12 / 16 / 73 / 2$

$14,450 \pm 100$

$\mathrm{NE}$ wall of trench, 22.5 to $23.2 \mathrm{~m}$ from reference point. Date is average; sample split as in Tx-1913: 14,210 $\pm 140,14,690 \pm 130$.

General Comment (SV,Jr): paleomagnetic directions and ${ }^{14} \mathrm{C}$ dates do not support existence of geomagnetic field excursion formerly reported in 14,500 yr mud at Tlapacoya (Liddicoat and Coe, 1974; Liddicoat $e t$ al, 1979). Tx-1784 believed contaminated by root hairs.

\section{North-Central Coastal Range series, Venezuela}

Samples from Holocene outcroppings along $\mathrm{N}$-central coast between Arrecifes and Borburata, DF, Venezuela. Coll to determine velocity of uplift of coastal range (Caribbean Mts). Coll 1975 and subm by C Schubert, Inst Venezolano Investigaciones Científicas, Caracas, Venezuela. Last number in sample title refers to elev in $\mathrm{m}$ above sea level.

Tx-2274. ARR-3, 0

$1170 \pm 80$

Conglomeratic beach rock, on Taguao Bay, ca $1.5 \mathrm{~km} \mathrm{~W}$ of Arrecifes $\left(10^{\circ} 33^{\prime} \mathrm{N}, 67^{\circ} 08^{\prime} \mathrm{W}\right)$.

Tx-2275. ARR-4, 0.5

$$
4480 \pm 50
$$

Coral, same general loc as Tx-2274.

Tx-2276. ARR-6, 0.5

$$
1020 \pm 60
$$
W). 
Tx-2277. ARR-10, 0.5

$970 \pm 70$ W).

Coral, on Taguao Bay, ca $1.8 \mathrm{~km}$ W of Arrecifes $\left(10^{\circ} 33^{\prime} \mathrm{N}, 67^{\circ} 08^{\prime}\right.$

Tx-2278. ARR-11, 0.5

Shell, same loc as $\mathrm{Tx}-2277$, above.

$1040 \pm 60$

Tx-2279. ARR-13, 0

Modern W).

Tx-2280. ARR-15, 0

$2840 \pm 80$

Conglomeratic beach rock, same loc as Tx-2279, above.

Tx-2281. ARR-18, 2

$3260 \pm 50$ $08^{\prime} \mathrm{W}$ )

Coral, on La Salina Bay, ca $3 \mathrm{~km} \mathrm{~W}$ of Arrecifes $\left(10^{\circ} 33^{\prime} \mathrm{N}, 67^{\circ}\right.$

Tx-2282. ARR-19, 0.5

Coral, same loc as Tx-2281, above.

$1860 \pm 60$

Tx-2283. ARR-20, 0.5

Coral, same loc as Tx-2281, above.

$1500 \pm 50$

Tx-2325. CH-2, 15-18

Coral, ca $2 \mathrm{~km} \mathrm{~W}$ of Puerto Colombia $\left(10^{\circ} 30^{\prime} \mathrm{N}, 67^{\circ} 38^{\prime} \mathrm{W}\right)$

Tx-2326. PAT-1, 0.5

$4500 \pm 80$ $\left.67^{\circ} 58^{\prime} \mathrm{W}\right)$

Coral, W side Patanemo Bay, ca $4.5 \mathrm{~km}$ E Puerto Cabello $\left(10^{\circ} 30^{\prime} \mathrm{N}\right.$,

Tx-2327. PAT-3, 1.2

Coral, W side Patanemo Bay $\left(10^{\circ} 30^{\prime} \mathrm{N}, 67^{\circ} 58^{\prime} \mathrm{W}\right)$.

$3620 \pm 90$

Tx-2328. PAT-5, 1.0

$3380 \pm 50$

Coral, same loc as $\mathrm{Tx}-2327$, above.

Tx-2329. BOR-2, 0

$3430 \pm 70$

Coral, W end Borburata beach, ca $3.5 \mathrm{~km}$ E Puerto Cabello $\left(10^{\circ} 30^{\prime}\right.$ $\left.\mathrm{N}, 67^{\circ} 59^{\prime} \mathrm{W}\right)$.

Tx-2330. BOR-5, 0

Shell, same loc as $\mathrm{Tx}-2329$, above.

$990 \pm 50$

Tx-2331. BOR-6, 0

Coral, same loc as Tx-2329, above.

$620 \pm 70$

Tx-2332. BOR-10, 1

$1310 \pm 60$

Coral, ca $300 \mathrm{~m} \mathrm{~W}$ Tx-2329, above.

General Comment (CS): velocity of uplift of Coastal Range varies between $0.054,0.061$, and $0.0033 \mathrm{~cm} / \mathrm{yr}$. However, real velocity of uplift probably 
higher because age of coral and shell fragments contained in beachrock may be considerably older than rock itself. For report, see Schubert et al (1977).

\section{PALEOBOTANICAL SAMPLES \\ Idaho, Washington}

\section{Hager Pond series, Idaho}

Peat samples from Core 1 (except as indicated) at Hager pond, mire ca $3.2 \mathrm{~km} \mathrm{~S}$ of Nordman, Idaho $\left(48^{\circ} 36^{\prime} \mathrm{N}, 116^{\circ} 57^{\prime} \mathrm{W}\right)$. Coll 1973 by $\mathrm{R} \mathrm{N}$ Mack and subm by V M Bryant, Anthropol Research Lab, Texas A \& M Univ, College Station, Texas. Figures in titles are depths in $\mathrm{cm}$ below surface.

Tx-2103. Hager Pond \#31, 913-916 $9110 \pm 110$ Core 2.

Tx-2104. Hager Pond \#32, 643-645

$$
\begin{aligned}
& 6980 \pm 480 \\
& 3030 \pm 110 \\
& 9510 \pm 190
\end{aligned}
$$

Tx-2105. Hager Pond \#33, 339-343

Tx-2108. Hager Pond \#36, 920-923

Core 2.

Tx-2110. Hager Pond \#38, 904-908

$8280 \pm 120$

Tx-2113. Hager Pond \#41, 279-283

$2940 \pm 80$

Tx-2115. Hager Pond \#43, 161-166

$2670 \pm 100$

Tx-2116. Hager Pond \#44, 600-605

$6630 \pm 80$

Tx-2119. Hager Pond \#47, 395-399

$4310 \pm 990$

Tx-2121. Hager Pond \#49, 500-505

$6390 \pm 230$

Tx-2673. Hager Pond C-1, 760-771

$7680 \pm 40$

Tx-2674. Hager Pond C-1, 805-810

$7960 \pm 310$

General Comment (RNM): Mazama ash is between Tx-2121 and Tx-2116. Dates confirm and define environmental events of last 10,000 yr or more accurate time scale than in previous analyses of this mire (Hansen, 1939; Rumley, 1956). For complete report, see Mack et al (1978a).

\section{Simpsons Flats series, Washington}

Samples from Core 7 in mire in closed depression at Simpsons Flats on Colville Indian Reservation, $45 \mathrm{~km} \mathrm{~N}$ of confluence of Sanpoil and Columbia Rivers $\left(48^{\circ} 25^{\prime} \mathrm{N}, 118^{\circ} 15^{\prime} \mathrm{W}\right)$. Coll 1973 and subm by $\mathrm{R} \mathrm{N}$ Mack, Dept Botany, Washington State Univ, Pullman, Washington. Figures in sample titles are depths in $\mathrm{cm}$ below surface.

Tx-2214. Simpsons Flats, 225-228 $1970 \pm 160$ Fibrous peat.

Tx-2215. Simpsons Flats, 264-268

$2630 \pm 160$ Gyttja. 
Tx-2216. Simpsons Flats, 340-344 Gyttja.

$$
3260 \pm 100
$$

Tx-2217. Simpsons Flats, 411-414 Gyttja.

$$
4120 \pm 170
$$

Tx-2218. Simpsons Flats, 477-480 Gyttja.

$$
4380 \pm 240
$$

Tx-2219. Simpsons Flats, 526-529

Gyttja.

$$
4790 \pm 230
$$

Tx-2220. Simpsons Flats, 632-635

Gyttja.

$$
5850 \pm 240
$$

Tx-2222. Simpsons Flats, 840-845 Wood.

$$
10,000 \pm 150
$$

Tx-2223. Simpsons Flats, 848 Wood.

Tx-2418. Simpsons Flats, 710 $9270 \pm 280$

Wood.

Tx-2470. Simpsons Flats, 730-735 Wood.

$9560 \pm 110$

General Comment (RNM): Mazama ash was at $680 \mathrm{~cm}$. Dates give rate of vegetational change with recession of glacial ice and subsequent climatic change. Sharp discontinuity between Tx-2418 and Tx-2220 suggests no preserved records for events between ca 9000 and $6700 \mathrm{yr}$. For complete report, see Mack, Rutter, \& Valastro (1978).

\section{Big Meadow series, Washington}

Gyttja from two cores from mire at Big Meadow on Meadow Creek, Pend Oreille Co, NE Washington $\left(48^{\circ} 43^{\prime} \mathrm{N}, 117^{\circ} 33^{\prime} \mathrm{W}\right)$. Coll 1972 and subm by $\mathrm{R} \mathrm{N}$ Mack. Numbers in titles refer to $\mathrm{cm}$ depths of samples in core. Samples listed in stratigraphic order from highest to lowest.
Tx-2203. Big Meadow, 76
$1170 \pm 100$
Tx-2295. Big Meadow, 149
$2480 \pm 130$
Tx-2416. Big Meadow, 253
$3050 \pm 70$
Tx-2414. Big Meadow, 307
$3390 \pm 100$
Tx-2415. Big Meadow, 363
$4210 \pm 110$
Tx-2073. Big Meadow, 563
$8910 \pm 80$
Tx-2417. Big Meadow, 581
$9130 \pm 320$ 
Tx-2226. Big Meadow, 607

General Comment (RNM): dates establish late Quaternary chronology of pollen record from Big Meadow and confirm relatively warmer climate than today between 9700 and 3330 BP. Present climatic conditions emerged ca 2400 BP. For complete report see Mack et al (1979).

\section{Waits Lake series, W ashington}

Gyttja core samples from Core $1, \mathrm{~S}$ shore Waits Lake ca $10 \mathrm{~m}$ from waterline, $2 \mathrm{~km} \mathrm{~W}$ of Colville R Valley, Washington $\left(48^{\circ} 11^{\prime} \mathrm{N}, 117^{\circ} 48^{\prime}\right.$ W). Subm to establish chronology for late Quaternary vegetational history in area. Coll 1972 and subm by R N Mack. Figures in titles are depths in $\mathrm{cm}$ below surface.

Tx-2657. Waits Lake, 125-135

$$
\begin{array}{r}
3530 \pm 80 \\
6250 \pm 100 \\
7910 \pm 100 \\
8480 \pm 260 \\
9880 \pm 150 \\
9980 \pm 160 \\
11,950 \pm 200
\end{array}
$$$$
\text { Tx-2658. Waits Lake, 275-294 }
$$$$
\text { Tx-2659. Waits Lake, 315-330 }
$$$$
\text { Tx-2660. Waits Lake, 440-456 }
$$$$
\text { Tx-2661. Waits Lake, 570-590 }
$$$$
\text { Tx-2662. Waits Lake, 660-684 }
$$$$
\text { Tx-2663. Waits Lake, 830-850 }
$$

General Comment (RNM): dates evidently unreliable in absolute sense since Tx-2660 and Tx-2659 bracket Mazama ash, dated elsewhere (Fryxell, 1965) at $6700 \mathrm{BP}$. Also Tx-2663 is above Glacier Peak ash, dated elsewhere (Mehringer et al, 1977) between 11,200 and 11,300 BP. Present dates probably about $1300 \mathrm{yr}$ old, due to contamination with older carbon. They provide useful within-the-core relative time frame. For complete report, see Mack et al (1978b).

\section{Mud Lake series, Washington}

Gyttja from two cores on NW margin of Mud Lake, $10 \mathrm{~km}$ NE Concunully, Washington $\left(48^{\circ} 34^{\prime} \mathrm{N}, 119^{\circ} 30^{\prime} \mathrm{W}\right)$. Numbers in title refer to cm depth in core. Coll 1972 and subm by $\mathrm{R} N$ Mack.

Tx-2686. Mud Lake, 254-260

$$
\begin{aligned}
8030 & \pm 150 \\
8840 & \pm 940 \\
9440 & \pm 120 \\
11,300 & \pm 560 \\
11,500 & \pm 560
\end{aligned}
$$$$
\text { Tx-2687. Mud Lake, 326-332 }
$$

Tx-2688. Mud Lake, 355-360

Tx-2689. Mud Lake, 424-466

Tx-2690. Mud Lake, 458-466

General Comment (RNM): series is below Mazama ash. For discussion, see Mack, Rutter, \& Valastro (1979).

\section{Bonaparte Meadows series, Washington}

Peat from two cores from middle of fen, Bonaparte Meadows, $28 \mathrm{~km}$ NW Republic, Washington $\left(48^{\circ} 54^{\prime} \mathrm{N}, 119^{\circ} 02^{\prime} \mathrm{W}\right)$. Coll 1972 and subm by R N Mack. Numbers in title refer to $\mathrm{cm}$ depths in core. 
Tx-2676. Bonaparte, 55-60

$1480 \pm 60$

Tx-2677. Bonaparte, 115-119

Tx-2678. Bonaparte, 150-155

$2170 \pm 220$

Tx-2679. Bonaparte, 190-195

$2440 \pm 100$

Tx-2680. Bonaparte, 245-250

$2570 \pm 70$

Tx-2681. Bonaparte, 305-310

$3340 \pm 90$

Tx-2682. Bonaparte, 350-355

$4780 \pm 240$

Tx-2683. Bonaparte, 410-415

$6750 \pm 190$

Tx-2882. Bonaparte, 415-421

$6790 \pm 130$

Tx-2881. Bonaparte, 435-439

$6810 \pm 190$

Tx-2883. Bonaparte, 468-473

$6870 \pm 110$

Tx-2884. Bonaparte, 492-497

$6930 \pm 110$

Tx-2684. Bonaparte, 525-530

$\mathbf{8 3 0 0} \pm \mathbf{8 0}$

Tx-2685. Bonaparte, 572-577

$9000 \pm 420$

$10,000 \pm 220$

General Comment (RNM): Tx-2676 to Tx-2683 are between St Helens W and Mazama ash. Tx-2882 immediately above Mazama ash. Tx-2881 and Tx-2883 between 2 Mazama ash units; confirms earlier field evidence that Mazama ash represents 2 separate eruptions (Mack, Okazaki, \& Valastro, 1979). Tx-2884, -2684, and -2685 below Mazama ash. For general discussion, see Mack, Rutter, \& Valastro (1979).

\section{Massachusetts}

\section{Tx-2948. Ned's Ditch 9, Massachusetts}

$3020 \pm 380$

Small twigs embedded in black and orange silt and fine sand, 305 to $310 \mathrm{~cm}$ below water surface, Core 9 , Ned's Ditch, center of oxbow lake of Connecticut $\mathrm{R}$ within city limits of Northampton, Massachusetts $\left(42^{\circ} 17^{\prime}\right.$ $30^{\prime \prime} \mathrm{N}, 72^{\circ} 37^{\prime} 30^{\prime \prime} \mathrm{W}$ ). Coll 1975 and subm by M H Sackett, Dept Biol Sci, Smith Coll, Northampton, Massachusetts. Comment (MHS): date is oldest obtained from oxbow. From same core, $349 \mathrm{~cm}$, was $\mathrm{Gx}-4378,710$ \pm 130 (Sackett, 1977, p 72); backcaving may have occurred at base of core.

\section{Granby Bog series, Massachusetts}

Peat samples from NE corner of Granby Bog, $0.8 \mathrm{~km} \mathrm{~S}$ of Bachelor Brook Rd, $9.3 \mathrm{~km}$ SSE of Amherst $\left(42^{\circ} 15^{\prime} \mathrm{N}, 72^{\circ} 30^{\prime} \mathrm{W}\right)$. Coll 1977 by Sackett and Sailor and subm by M H Sackett.

Tx-2945. Granby D-14.

902 to $927.4 \mathrm{~cm}$ depth; near base of Pollen Zone Cl.

$$
8520 \pm 200
$$

Tx-2946. Granby D.21

1205 to $1230 \mathrm{~cm}$ depth; Pollen Zone A4.

$10,300 \pm 370$ 
General Comment (MHS): chronology of Granby Bog agrees well with other ${ }^{14} \mathrm{C}$-dated pollen diagrams in S New England (Beetham and Niering, 1961; Flint and Deevey, 1951; Totoket series in R, 1959, v 1, p 146-147).

\section{ARCHAEOLOGIC SAMPLES}

\section{Texas}

\section{Tx-3173. Castner Range}

$$
3080 \pm 160
$$

Charcoal from Castner Range site (31:106:3:75), E side of Franklin Mt, El Paso, Texas ( $\left.31^{\circ} 52^{\prime} 46^{\prime \prime} \mathrm{N}, 106^{\circ} 25^{\prime} 45^{\prime \prime} \mathrm{W}\right)$. From ash lens ca $35 \mathrm{~cm}$ below surface; late San Pedro Cochise phase. Coll 1978 by M Thompson and subm by L Herrington, Texas Hist Comm, Austin, Texas. Comment (LH): agrees with other dates of comparable cultural assignment in Hueco Bolson (Thompson \& Beckett, 1979).

\section{McDonald site series}

Charcoal samples from hearth, $87 \mathrm{~cm}$ below surface, early component of McDonald site (X41HI171), E bank of Hackberry Creek, $2.1 \mathrm{~km}$ WNW of Vaughn, Texas, in Aquilla Reservoir basin (31 $55^{\prime} 12^{\prime \prime} \mathrm{N}, 97^{\circ} 11^{\prime} 37^{\prime \prime}$ W). Coll 1978 and subm by S A Skinner, Archaeol Research Prog, Southern Methodist Univ, Dallas.

\section{Tx-3168. X41HI171/23/1}

$$
\begin{array}{r}
780 \pm 70 \\
1010 \pm 70
\end{array}
$$

Tx-3169. X41HI171/23/2

General Comment (SAS): dates not in $1 \sigma$ agreement. Component includes Coles Creek Incised sherd; Tx-3169, agreeing with late part of date range usually assigned to this type, is probably nearer actual age than Tx-3168.

\section{George C Davis site}

Samples from George C Davis site (41CE19), early Caddo site E side Neches R valley, $9.6 \mathrm{~km}$ SW of Alto, Cherokee Co, Texas $\left(31^{\circ} 35^{\prime} \mathrm{N}, 95^{\circ}\right.$ $\left.10^{\prime} \mathrm{W}\right)$. Previous series from site are in R, 1970, v 12, p 626-629, and R, 1975 , v 17, p 71-76. For extensive review of ${ }^{14} \mathrm{C}$ dating of site, see Story and Valastro (1977).

\section{George $C$ Davis village series}

Samples from hitherto uninvestigated part of site, NE of previously investigated portions. Coll 1978 and subm by D Creel, Dept Sociol \& Anthropol, Texas A \& M Univ, College Station. "Corrected" ages are corrected for $\delta^{13} \mathrm{C}$ and dendrochronology (Damon et al, 1974).

Tx-3267. 41CE19/237

$$
\begin{array}{r}
\mathbf{5 6 0} \pm \mathbf{6 0} \\
\delta^{13} C=-11.8 \% \text { 。 }
\end{array}
$$

Charred corn cob cupules and fragments, Unit 43, Feature 184-3. Corrected, $794 \pm 66$.

Tx-3268. 41CE19/242

$$
1040 \pm 230
$$$$
\delta^{13} \mathrm{C}=-26.9 \%
$$

Woody charcoal from bottom of pit, Unit 45, Feature 185-4. Corrected, $984 \pm 232$. 
Tx-3269. 41CE19/244A

Woody charcoal, Unit 44, Feature 186-1. Corrected, $700 \pm 80$.

Tx-3270. 41CE19/244B

$\mathbf{7 7 0} \pm \mathbf{7 0}$

Charred corn cob cupules, Unit 44, Feature 186-1. Corrected, $970 \pm$ 80.

Tx-3271. 41CE19/246A

$\mathbf{7 9 0} \pm 80$

Woody charcoal, Unit 46, Feature 187-3. Corrected, $748 \pm 89$.

Tx-3272. 41CE19/247

$780 \pm 100$

Woody charcoal, Unit 46, Feature 187-5. Corrected, $765 \pm 107$.

Tx-3273. 41CE19/250A

$950 \pm 80$

Woody charcoal, Unit 48, Feature 189-6. Corrected, $930 \pm 89$.

Tx-3274. 41CE19/250B $\mathbf{4 4 0} \pm \mathbf{9 0}$
$\delta^{13} \mathrm{C}=-10.4 \%$
Corrected, $700 \pm$ 104.

Charred corn cob cupules, Unit 48, Feature 189-6.

Tx-3275. 41CE19/254A

Woody charcoal, Unit 45, Feature 185-11. Corrected, $883 \pm 98$.

$910 \pm 90$

$\delta^{13} \mathrm{C}=-26.4 \%$

Tx-3276. 41CE19/254B $\mathbf{5 5 0} \pm \mathbf{6 0}$
$\delta^{13} C=-10.1 \% 0$
Corrected, $802 \pm$ 66.

Charred corn cob cupules, Unit 45, Feature $185-11$. Corrected, $802 \pm$

Tx-3307. 41CE19/matrix sample 468

$880 \pm 110$

Charred corn cob cupules, Unit 48, Feature 189-1. Corrected, $1099 \pm$ 117.

Tx-3308. 41CE19/253B

$620 \pm 100$

Charred hickory nut shell, Unit 43, Feature 184-4. Corrected, $625 \%$ 104.

Tx-3309. 41CE19/238

$1170 \pm 100$

Charred hickory nut fragments, Unit 45 , Feature 18 $1215 \pm 105$

Tx-3310. 41CE19/239

$610 \pm 100$

Charred corn cob cupules, Unit 45, Feature 185-1. Corrected, $828 \pm$ 104. 
Tx-3311. 41CE19/246B

$$
1140 \pm 160
$$

$\delta^{13} C=-26.6 \%$

Probable charred cane, Unit 46, Feature 187-3. Corrected, $1100 \pm 163$.

\section{Tx-3312. 41CE19/253A}

$$
1190 \pm 80
$$

Probable charred cane, Unit 43, Feature 184-4. Corrected, $1128 \pm 87$.

General Comment (DC): all samples except Tx-3268 are from small charcoal-filled features which may be smudging pits of short-term use. Dates generally agree with previous dating of early Caddoan occupation at Davis site (Story \& Valastro, 1977) and appear to relate mostly to later part of this occupation, although there is some indication of early occupation as well.

\section{George C Davis F 193-1 series}

Wood charcoal from fill of pit, Feature 193-1, in Unit 62, W of Mound C, George C Davis site. Coll 1978 and subm by P Thurmond and U Kleinschmidt, Texas Archeol Research Lab, Univ Texas, Austin.

Tx-3399. Davis F193-1, 299

$$
990 \pm 60
$$

Zone 4.

\section{Tx-3400. Davis F193-1, 255}

$$
1020 \pm 60
$$

\section{Zone 5.}

Tx-3401. Davis F193-1, 288

$800 \pm 40$

\section{Zone 10 (deepest).}

General Comment (PT, UK): pit fill was distinctly stratified, but matching sherds from different zones show that deposition was all one event. Occurrence of youngest date (Tx-3401) in deepest zone supports this conclusion. If Tx-3401 represents date of deposition, event was late in occupation of site.

\section{Arkansas}

\section{Amos site series}

Samples from Late Caddoan component, Amos site (3MN62), Upper Caddo Valley, $0.4 \mathrm{~km} \mathrm{SW}$ of Caddo Gap, Montgomery Co, Arkansas (34 $\left.23.5^{\prime} \mathrm{N}, 93^{\circ} 37.5^{\prime} \mathrm{W}\right)$. Coll 1976 by F Schambach and subm by A M Early, Arkansas Archeol Survey, Henderson State Univ, Arkadelphia, Arkansas.

\section{Tx-2858. 76-690-62}

$\mathbf{2 3 0} \pm \mathbf{5 0}$

Woody twigs from Feature 4, small pit filled with twigs, nut hulls, and possible cultigens. Unit N18W42, 35cm depth. Comment (AME): date too recent to refer to Late Caddoan component.

Tx-2859. 76-690-127-1

$440 \pm 70$

Wood charcoal from concentration of organic refuse on perimeter of Feature 3, Unit N16W44. Comment (AME): date appropriate for Late Caddoan component. 


\section{Boydell site series}

Carbonized $\log$ samples from Boydell site (3AS58), Bartholomew phase Plaquemine, E side Bayou Bartholomew, in Boydell, Arkansas $\left(30^{\circ}\right.$ $22^{\prime}$ N, $91^{\circ} 29^{\prime} \mathrm{W}$ ). Coll 1977 and subm by P Stacy, Arkansas Archeol Survey, Univ Arkansas, Monticello.

\section{Tx-2885. Boydell 77-764-1}

$S$ central part of mound, $1.2 \mathrm{~m}$ below surface; Feature 1.

\section{Tx-2886. Boydell 77-764-27}

$\mathrm{N}$ side Feature 2, ca $2 \mathrm{~m}$ below surface.

\section{Tx-2887. Boydell 77-764-41}

W side Feature 2, ca $2 \mathrm{~m}$ below surface, with Burial 5 .

$$
690 \pm 60
$$

$$
750 \pm 80
$$

General Comment (JM House): dates help confirm Rolingson's (1976, p 119) estimate of AD 1200 to 1400 for Bartholomew Phase of Plaquemine, and strengthen proposed chronologies for Winterville (Brain, 1969, p 279282), lower Yazoo (Phillips, 1970, p 955-961), and Tensas (Hally, 1966).

\section{TX-3248. 34SM-20/\#2}

\section{Oklahoma} Rogers Rite (34Sm-20), on ridge N side Little R, ca 4.8km NW Sasakwa, Seminole Co, Oklahoma (34 $\left.59^{\prime} 70^{\prime \prime} \mathrm{N}, 96^{\circ} 35^{\prime} 60^{\prime \prime} \mathrm{W}\right)$. Component A, Plains Woodland. Coll 1972 and subm by R Drass, Oklahoma Archaeol Survey, Norman. Comment (RD): date as expected; agrees with UGa$2510,1310 \pm 105$, from same component (Drass, pers commun).

\section{Blessingame site series}

Samples from Blessingame site (34Pu-74), Archaic and early Caddoan site in Jackfork Valley $5.6 \mathrm{~km} \mathrm{~N}$ of Clayton, in Clayton Reservoir basin,

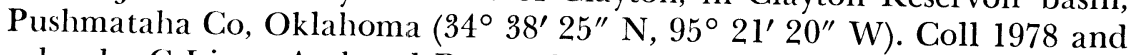
subm by Ci Lintz, Archaeol Research \& Management Center, Univ Oklahoma, Norman. UGa dates cited are from Lintz (pers commun). Comments by CL.

Tx-3280. 34Pu-74, \#2 $1010 \pm 50$

Charred wood from roof fall, Structure 1, Sample C-6, Level 3, Sq B35-17. Early Caddo. Comment: sample pretreated at Texas lab, split, half-dated at Univ Georgia: UGa-2721, $890 \pm 65$. Georgia date not inconsistent with present date; both dates consistent with early Caddo materials. Another date from same structure is UGa-2532, $185 \pm 60$, anomalously young. See also Tx-3283, below.

Tx-3281. 43Pu-74, \#4

Charred wood, roof fall post, Structure 2, Level 2, Sq C64-11. Early Caddo. Comment: sample pretreated at Texas lab, split, half-dated at Univ Georgia: UGa-2720, $790 \pm 65$, later than present date, but both dates consistent with early Caddo assignment. Another date from same structure is UGa-2533, $565 \pm 60$, anomalously young. 
Tx-3282. 43Pu-74, \#8

$500 \pm 40$

Charred wood from fill of Feature 78-9, rock hearth, Level 2, S half Sq A21-18. Comment: UGa-1518, $220 \pm 100$, from same feature; dates do not agree.

Tx-3283. 43Pu-74, \#7

$\mathbf{5 3 0} \pm \mathbf{5 0}$

Charred cane from roof fall, Structure 1, Level 4, Sqs B35-11, B35-9, B35-10, B35-12. Comment: date significantly younger than other dates from this structure (see comment for Tx-3280, above). Lab Comment: no $\delta^{13} \mathrm{C}$ determination made. Anomalous date possibly due to fractionation in cane; our experience is that some species of cane fractionate, others do not. Present sample not identified botanically.

\section{Tx-3284. 43Pu-74, \#10}

$1920 \pm 60$

Charred wood, probably assoc with Feature 78-6, rock hearth, Level $2(10$ to $20 \mathrm{~cm})$, Sq A20-14. Comment: date inexplicably early; stratigraphically equivalent to Feature 78-9 (Tx-3282, above, and UGa-1518, $220 \pm 100)$ and is stratigraphically above UGa-2534, $175 \pm 175$.

\section{Parker site series}

\section{North Carolina}

Charcoal samples from Parker site (31Dv4), late Woodland site $250 \mathrm{~m}$ $\mathrm{S}$ of Yadkin R on Horseshoe Neck, ca $11 \mathrm{~km}$ NW of Churchland, North Carolina $\left(35^{\circ} 50^{\prime} \mathrm{N}, 80^{\circ} 31^{\prime} \mathrm{W}\right)$. Coll 1972 and subm by J N Woodall, Mus of Man, Wake Forest Univ, Winston-Salem, North Carolina.

Tx-2818. Parker A

$970 \pm 80$

From postmold, Sq N95/E98, Level 3, 30 to $39 \mathrm{~cm}$ below surface.

Tx-2819. Parker B

$1340 \pm 60$

Sq N78/E100, Level 3, 30 to $45 \mathrm{~cm}$ below surface.

Tx-2820. Parker C

$120 \pm 260$

Trash pit in Sq N95/E100, 99.33 to $99.36 \mathrm{~m}$ above datum. Pit had late Woodland sherds and points, 2 late Archaic points, mussel shells, animal bones.

General Comment (JNW): Tx-2818 and -2819 agree with evidence of Late Archaic point assocs that net-impressed pottery (and large Yadkin R sites with this ware) is not protohistoric in age. Reason for late date of Tx-2820 not apparent, but fill of trash pit was dark and intrusions might have been present without being detected.

\section{Ohio}

\section{Tx-2374. Eddie Paul Rockshelter}

$$
\mathbf{6 6 0} \pm 50
$$

Eddie Paul Rockshelter, on top of hill W of Stump Run, $1.6 \mathrm{~km} \mathrm{~S}$ of Frazeysburg, Ohio $\left(40^{\circ} 05^{\prime} \mathrm{N}, 82^{\circ} 07^{\prime} \mathrm{W}\right)$. Earliest occupation of site, assoc with Late Woodland occupation including triangular projectile points and Peters cord-marked pottery. From $0.55 \mathrm{~m}$ depth, lowest occupation level. Coll 1972 by J Carskadden and subm by J P Morton, Dept 
Geol Sci, Univ Texas, Austin. Comment (JPM): date later than expected; should be earlier than Philo phase (see Richards site series, below) which has shell-tempered pottery. Reason for late date unknown.

Tx-2462. Merry's Cave $2290 \pm 50$

Charcoal from Merry's Cave site, on Salt Creek, $1.6 \mathrm{~km} \mathrm{~N}$ of junction with White Eyes Creek, $8 \mathrm{~km} \mathrm{WNW}$ of Chandlersville, Ohio $\left(39^{\circ} 55^{\prime} \mathrm{N}\right.$, $81^{\circ} 52^{\prime} \mathrm{W}$ ). From layer D-3, lowest level where pottery occurs: Early Adena. Coll 1969 by J Carskadden and subm by J P Morton. Comment (JPM): date recent for Early Adena.

\section{Richards site series}

Richards site (HA-13), Middle Fort Ancient aspect, Philo phase, on $\mathrm{N}$ bank Muskingum R, $0.8 \mathrm{~km} \mathrm{~W}$ of Duncan Falls $\left(39^{\circ} 52^{\prime} \mathrm{N}, 81^{\circ} 55^{\prime} \mathrm{W}\right)$. Coll 1975 by Carskadden and subm by J P Morton.

Tx-2344. Richards HA-13-A $660 \pm 60$

Bottom of Fire Pit A, $0.76 \mathrm{~m}$ below plow zone; assoc with Philo Punctate pottery.

\section{Tx-2345. Richards HA-13-B}

$$
680 \pm 50
$$

Bottom of Fire Pit B, $0.6 \mathrm{~m}$ below plow zone.

\section{Tx-2346. Richards HA-13-C}

Bottom of Fire C, $0.76 \mathrm{~m}$ below plow zone.

$$
600 \pm 70
$$

General Comment (JPM): dates consistent with previous estimates for age of Philo phase.

\section{Hilltop Mound Group series}

Hilltop Mound Group (HA-3), Adena and Late Hopewell site on $\mathrm{N}$ bank of Muskingum R, on ridge above Richards site $0.8 \mathrm{~km} \mathrm{~W}$ of Duncan Falls $\left(39^{\circ} 52^{\prime} \mathrm{N}, 81^{\circ} 55^{\prime} \mathrm{W}\right)$. Coll 1975 by $\mathrm{J}$ Carskadden and subm by J P Morton.

\section{Tx-2347. Hilltop Mounds, HA-3D-1}

$$
2990 \pm 80
$$

Mound D, Feature 1, central fire basin on primary mound, Mound D. Comment (JPM): most assocs are Late Hopewell, but Early Woodland pottery was nearby; date indicates Early Woodland.

\section{Tx-2373. Hilltop Mounds, HA-3B-1}

$1650 \pm 60$

Charred $\log$ in fire basin on floor of primary mound, Mound $B$. Comment (JPM): date consistent with Late Hopewell.

Tx-2375. Hilltop Mounds, HA-3E-1

From living area of House E, Late Adena. Comment (JPM): date consistent with Late Adena and Early Hopewell.

\section{Washington}

\section{Tx-3304. Miller site \#55, Washington}

$$
2400 \pm 110
$$

Charcoal fragments from Miller site (45FR5) on Strawberry I, in Snake R, $6.4 \mathrm{~km}$ upstream from its mouth, Franklin Co, Washington $\left(46^{\circ}\right.$ 
$\left.14^{\prime} \mathrm{N}, 118^{\circ} 59^{\prime} \mathrm{W}\right)$. Side of $43.5 \mathrm{E}$ wall, $59.4 \mathrm{~S}$ to $60.0 \mathrm{~S}$, alt 103.60 to $103.70 \mathrm{~cm}$. Coll 1978 and subm by R R Mierendorf, Washington State Univ, Pullman, Washington. Comment (RRM): date assoc with nonhousepit occupation; underlies large housepit occupation.

\section{Tx-3066. Iron Jaw Wilcox Burial}

Wood charcoal from Iron Jaw Wilcox Burial (24RB93), on first alluvial terrace above Iron Jaw Creek, ca $6.4 \mathrm{~km} \mathrm{~S}$ of Hathaway, Montana $\left(46^{\circ} 13^{\prime} 38^{\prime \prime} \mathrm{N}, 106^{\circ} 13^{\prime} 11^{\prime \prime} \mathrm{W}\right)$. Above and among bones of primary burial, 2 to $30 \mathrm{~cm}$ subsurface. Coll 1978 by J Clark and subm by R A Teegarden, U S Bur Land Management, Miles City, Montana. Comment (RAT): no grave goods assoc; ${ }^{14} \mathrm{C}$ date allows placement of burial in time.

\section{Benson's Butte series}

Charcoal from hearths and bone from rock-walled dwelling in Benson's Butte site (24BH1726; Fredlund, 1979). Late Prehistoric site on spring fork of Young's Creek, tributary of Tongue R, Crow Indian Reservation, Big Horn Co, Montana, ca $32 \mathrm{~km} \mathrm{~N}$ of Sheridan, Wyoming $\left(45^{\circ}\right.$ $\left.01^{\prime} 34^{\prime \prime} \mathrm{N}, 107^{\circ} 01^{\prime} 13^{\prime \prime} \mathrm{W}\right)$. Coll and subm 1977, 1978 by L B Fredlund, Montana Tech Alumni Foundation, Mineral Research Center, Butte, Montana. For previous dates, see R, 1977, v 19, p 317. Cm figures are depths below surface.

Tx-2795. Benson's Butte \#6

$940 \pm 60$

Charcoal, Feature 16,35 to $40 \mathrm{~cm}, 1 S 38 \mathrm{~W}$.

Tx-2796. Benson's Butte \# 7

$1510 \pm 60$

Charcoal, Feature 18,40 to $50 \mathrm{~cm}, 2 \mathrm{~N} / 3 \mathrm{~N} 37 \mathrm{~W} / 38 \mathrm{~W}$.

Tx-2797. Benson's Butte \#8

$4230 \pm 50$

Charcoal, Feature 20,60cm, 6 N $38 \mathrm{~W}$.

Tx-2798. Benson's Butte \#9

$1140 \pm 50$

Charcoal, Feature 20, 40 to $50 \mathrm{~cm}, 6 \mathrm{~N} 38 \mathrm{~W}$.

Tx-3111. Benson's Butte \# 10

$1000 \pm 90$

Bone, Feature 19, 40 to $60 \mathrm{~cm}$, various locations.

Tx-3112. Benson's Butte \#11

$1770 \pm 200$

Bone, Feature 19, 40 to $60 \mathrm{~cm}$, various locations.

General Comment (LBF): charcoal dates fit well within expected range of early Late Prehistoric period, except for Tx-2797, which is inexplicably early. Bone date, Tx-3111, consistent with major occupation of site; however, Tx-3112 thought to be more realistic date for structure as living surface is stratigraphically below latest occupation. For further details see Fredlund (1979). 


\section{Hatwai site series}

\section{Idaho}

Charcoal from Hatwai site (10NP143), at confluence of Hatwai Creek and Clearwater $\mathrm{R}$, ca $7 \mathrm{~km} \mathrm{E}$ of Lewiston, Idaho $\left(46^{\circ} 26^{\prime} 30^{\prime \prime} \mathrm{N}, 116^{\circ} 54^{\prime}\right.$ 00" W). Coll 1978 by Green, Ames, and others and subm by J P Green and $\mathrm{K}$ M Ames, Dept Sociol, Anthropol, and Ciriminal Justice Admin, Boise State Univ, Boise, Idaho. Comments by JPG and KMA.

\section{Tx-3081. 10NP143.77}

$$
9320 \pm 1830
$$

Very small samples from remnant sand layer over alluvial bar gravel, 9.6-11.8N/90-91E, alt 232.6 to $232.8 \mathrm{~cm}$. Early Alluvium (Qae) (Hammett, 1976). Comment: dates overbank sand deposition in broad meander at midpoint bar position. Stratigraphically below Tx-3082 and -3083 , below.

\section{Tx-3082. 10NP143-78}

$8560 \pm 520$

Truncated surface under upper gravel, $10-15 \mathrm{~N} / 89-90 \mathrm{E}$, alt 232 to $232.6 \mathrm{~cm}$. Early Alluvium (Qae). Comment: dates upper limit for truncation of overbank chute sediments and start of period of increased stream competence. Dates same episode as WSU-1828, 6165 \pm 210 (Ames, pers commun); present date in better agreement with geologic evidence. Stratigraphically above $\mathrm{Tx}-3083$, below.

\section{Tx-3083. 10NP143-17/78}

$$
9280 \pm 110
$$

In overbank channel, $13-15 \mathrm{~N} / 89-90 \mathrm{E}$, alt $321.8 \mathrm{~m}$. Mid-point bar location. Comment: dates start of overbank chute deposition; dates upper limit for truncation of underlying deposits. Geologically same as WSU1840, $7860 \pm 90$ (Ames, pers commun); present date in better agreement with geologic evidence. Early Alluvium (Qae). Unconformably overlies Tx-3081; underlies Tx-3082.

\section{Tx-3084. 10NPI43-87}

Bottom of channel cut into Early Alluvium, 13-14N/259E, alt 231.85 to $231.90 \mathrm{~cm}$. Comment: dates upper limit for cut. Dates cut and fill episode represented by Overbank "A" set, chute fill. Overbank episode records vigorous Clearwater $\mathrm{R}$ flow in Altithermal period. Upper point bar location; correlates with units at mid and lower bar positions.

\section{Tx-3085. 10NP143-102}

Organic fraction of Overbank Laminae "C" set, Unit B, 12.5-13.6N/ $259 \mathrm{E}$, alt 232.78 to $232.81 \mathrm{~m}$. Comment: dates episode of increased Clearwater $\mathrm{R}$ flow, roughly co-eval with Tucannon phase pit houses ( $\mathrm{T} x-3088$, -3263 , below). Upper point bar location, correlates with units at mid and lower bar positions. Middle Alluvium unit (Qam).

\section{Tx-3086. 10NP143-64}

$9160 \pm 230$

Occupational surface in Early Alluvium (Qae) unit, 9-12N/56-60E alt 231.9 to $232.1 \mathrm{~cm}$. Comment: laterally equivalent to $\mathrm{Tx}-3083$, above; dates agree. Dates upper limit for lower point bar gravels containing Windust phase artifacts (Leonhardy and Rice, 1970). 


\section{Tx-3088. 10NP143-103}

Small branch or house timber, lower floor House 2; 15.35-15.40N/ 141.18-141.40E, alt $233.25 \mathrm{~cm}$. Comment: pit house of Tucannon phase (Leonhardy and Rice, 1970); below upper floor of house, from which came Tx-3092, -3264, below.

Tx-3089. 10NP143-70A

$$
360 \pm 60
$$

From intrusive pit, Feature 90, dug into House 3, Tucannon phase pit house, $2.05-3.05 \mathrm{~N} / 59.45 \mathrm{E}$, alt $234 \mathrm{~cm}$. Comment: dates trash in pit.

Tx-3090. 10NP143-4A-5

$150 \pm 70$

From pit, Feature 2, 26.20-27.25S $/ 32 \mathrm{~W}$, alt $234 \mathrm{~cm}$. Comment: small side-notched points assoc; latest precontact date for site use.

Tx-3091. 10NP143-37

$2270 \pm 150$

Buried soil surface on upper Tucannon sediment, $18-28 \mathrm{~S} / 32 \mathrm{~W}$, alt 232.6 to $232.7 \mathrm{~cm}$. Comment: Middle Alluvium; composite sample from along contact, Qal position; correlates with paleosol developed on Qam.

Tx-3092. 10NP143-119

$\mathbf{3 4 2 0} \pm \mathbf{3 8 0}$

Composite sample from small area on upper floor House 2, 14-16N/ $141-145 \mathrm{E}$, alt 233.4 to $233.5 \mathrm{~cm}$. Comment: assoc with $\mathrm{Tx}-3264$, below; dates agree closely. Appropriately later than Tx-3088, above, on lower floor of same house. See Tx-3264, below, for further comments.

Tx-3158. 10NP143-217

$\mathbf{9 8 5 0} \pm \mathbf{8 7 0}$

Intergravel sand lens at lower point bar position; $10-12 \mathrm{~N} / 56-60 \mathrm{E}$, alt 230.94 to $231.38 \mathrm{~cm}$. Comment: assoc with Windust phase artifacts, postMissoula flood gravels. Stratigraphically inconsistent with Tx-3159, below; present date probably too recent.

Tx-3159. 10NP143-187

$10,820 \pm 140$

Lower point bar gravel unit, 8-10N/51.3-54.0E. Comment: Windust phase artifacts directly assoc; first post-Missoula flood occupation. Overlies Tx-3158, above, which has more recent date; present date believed more reliable because consistent with those from Marmes Rock Shelter for initial occupation (Rice, 1972).

Tx-3160. 10NP143-216

$10,110 \pm 720$

Intergravel sand lens, $14-16 \mathrm{~N} / 124-127 \mathrm{E}$, alt 232.64 to $233.03 \mathrm{~cm}$. Midpoint bar position. Comment: dates period of high stream competence. Artifacts in overlying gravel foreset unit.

Tx-3161. 10NP143-283

$5450 \pm 120$

Organic fraction of Overbank Lamina "B", from buried channel at intersection of lower point bar and creek; $0.5-1.0 \mathrm{~N} / 48-50 \mathrm{E}$, alt 231 to $231.38 \mathrm{~cm}$. Comment: dates episode of increased stream competence. Gives median age for ashy (Mazama) fan deposits derived from Hatwai Creek. 
Tx-3262. 10NP143-261

$5550 \pm 220$

Composite sample from fill of large pit intrusive into fill of House 1; 1.0-12N/128-130E, alt 233 to $233.1 \mathrm{~cm}$. Comment: stratigraphically more recent than Tx-3263, below, and WSU-1842, $3330 \pm 70$; present date must be too old for age of pit.

Tx-3263. 10NP143-271

Composite sample from floor of House 1, 10-12N/128-130E, alt 232.9 to $233 \mathrm{~cm}$. Comment: agrees with Tx-3088, above, in dating first pit house here, and provides support in dating House 5 at Alpowai site (Brauner, 1976). Hatwai and Alpowai are thus earliest villages on Columbia Plateau.

\section{TX-3264. 10NP143-186}

Composite sample from small pit assoc with upper floor of House 2; $14.7-15.4 \mathrm{~N} / 142.18-143.05 \mathrm{E}$, alt 232.82 to $233.20 \mathrm{~cm}$. Comment: agrees closely with Tx-3092, above. These 2 dates, and dates on House 1 (WSU$1842,3330 \pm 70$; WSU-1878, $3130 \pm 90$; Ames, pers commun) and House 4 (WSU-1892, $3240 \pm 90$; Ames, pers commun) date second major house construction episode at site, Tucannon phase.

\section{Tx-3265. 10NP143-117}

$\mathbf{8 8 0 0} \pm 1310$

Small composite sample on occupational surface in late glacial overbank sediment; $7-12 \mathrm{~N} / 51-60 \mathrm{E}$, alt $231.84 \mathrm{~cm}$. Comment: assoc with early Cascade phase material. Early Alluvium (Qae). Duplicate sample of Tx-3086, above, with which it agrees.

\section{Tx-3266. 10NP143-229}

$8660 \pm 1660$

Small composite sample assoc with upper gravel veneer on lower point bar, Feature 63, 6-12N/51-60E, variable alt. Comment: Windust phase cultural material assoc. Laterally equivalent to episode dated by Tx-3082; dates agree.

General Comment on Hatwai series: series provides first good set of dates for an open Windust phase site, establishes initiation of pit house villages on Columbia Plateau at 4300 to $4000 \mathrm{BP}$, and provides chronologic base for developing Clearwater $\mathrm{R}$ alluvial sequence.

\section{Curecanti Project, Colorado}

Samples from sites in Curecanti area, Blue Mesa Reservoir Basin, on Gunnison R, W of Gunnison, Colorado. Coll 1978 by R T Euler and subm by M Steiger, Midwest Archeol Center, Lincoln, Nebraska.

\section{GN191 series}

Charcoal from Site 5GN191, extensive lithic deposit with slab-lined firepits, points including variant type of Pinto Basin; $S$ bank of Gunnison $\mathrm{R}$, ca $22.5 \mathrm{~km} \mathrm{~W}$ of Gunnison $\left(38^{\circ} 28^{\prime} \mathrm{N}, 107^{\circ} 08^{\prime} \mathrm{W}\right)$.

Tx-3149. \#19

Feature 11, unlined firepit. 
Tx-3152. \#16

Feature 3, slab-lined firepit.

Tx-3155. \#18

Feature 6, slab-lined firepit.

General Comment (MS): dates earlier than expected; all are before end of Altithermal.

\section{GN205 series}

Charcoal from site 5GN205, in Elk Creek development area, ca $0.8 \mathrm{~km}$ $\mathrm{N}$ of Blue Mesa Reservoir, ca $24 \mathrm{~km} \mathrm{~W}$ of Gunnison $\left(38^{\circ} 28^{\prime} \mathrm{N}, 107^{\circ} 10^{\circ}\right.$ W). All samples are from same stratigraphic level, 20 to $30 \mathrm{~cm}$ below present ground surface.

Tx-3150. \#420/422

$4430 \pm 300$

Feature 1, circular ash stain (probable habitation structure), general fill, ca $3 \mathrm{~m}$ diam.

Tx-3151. \#443

$4560 \pm 80$

From hearth on edge of in situ lithic concentration.

Tx-3154. \#297/298

$$
9800 \pm 830
$$

Same location as Tx-3151, above.

Tx-3156. \#442

$$
7060 \pm 110
$$

From poorly defined charcoal stain, $4 \mathrm{~m}$ NW of Feature 1 .

Tx-3157. \#437

$4270 \pm 90$

Sample from possible cribbing from Feature 1.

General Comment (MS): dates from Feature 1 consistent; earlier than expected.

Tx-3153. 5GN200, Catalog \# 1

$4520 \pm 120$

Charcoal from Site 5GN200, within Elk Creek development area (see 5GN205 series, above). From possible shallow hearth in test pit. Comment (MS): consistent with most other dates from similar components in Curecanti area.

\section{Perijá series, Venezuela}

\section{South America}

Charcoal samples from 2 sites in Perijá Dist, state of Zulia, Venezuela. Coll 1976 and subm by E Wagner, Dept Antropol, IVIC, Caracas, Venezuela.

Tx-2608. Perijá, Caño Pescado

$9140 \pm 140$

From Trench A, level 0.25 to $0.5 \mathrm{~m}$, Caño Pescado site, on riverbank where Caño Pescado enters Rio Palmar $\left(10^{\circ} 41^{\prime} \mathrm{N}, 72^{\circ} 26^{\prime} \mathrm{W}\right)$.

\section{Tx-2609. Perijá, Berlin \# 1}

$490 \pm 70$

Trench V, surface to $0.25 \mathrm{~m}$, Berlin site, in valley between Caño Pescado and Caño Colorado, ca $1.5 \mathrm{~km}$ from Rio Palmar $\left(10^{\circ} 38^{\prime} \mathrm{N}, 72^{\circ}\right.$ $25^{\prime} \mathrm{W}$ ). 
Tx-2610. Perijá, Berlin \#2

Same as Tx-2609, level 0.25 to $0.5 \mathrm{~m}$.

Tx-2611. Perijá, Berlin \#3

Same as Tx-2609, Level 0.5 to $1 \mathrm{~m}$.

$8420 \pm 970$

General Comment (EW): assoc ceramics are similar to those at El Diluvio site (Tx-2409 to -2413; R, 1978, v 20, p 265), which were widely varied. Present dates much too old to date occupations, except Tx-2609 which is too young. Samples seem to consist partially of coal from Tertiary formations of Perijá area.

\section{La Galgada series, Peru}

Carbonized wood from pre-ceramic temples, La Galgada site, $1 \mathrm{~km} \mathrm{~S}$ of La Galgada village on E bank of Rio Tablachaca, Prov Pallasca, Peru $\left(8^{\circ} 28^{\prime} \mathrm{S}, 78^{\circ} 9^{\prime} \mathrm{W}\right)$. Coll 1978 and subm by T Grieder, Art Dept, Univ Texas, Austin.

\section{Tx-3166. C1-J6}

Fire pit in Floors 3, 5, 7, Sec C1, Sq J6.

Tx-3167. D1-D3

Fire pit in Floor 25, Sec D1, Sq D3.

$3660 \pm 80$

General Comment (TG): dates provide rough time range for pre-ceramic temples.

\section{Vegas series, Ecuador}

Shell (Anadara tuburculosa) and human bone from Vegas culture type site midden, on low hill 1km SW Santa Elena, Santa Elena Peninsula, Prov Guayas, Ecuador ( $\left.02^{\circ} 13^{\prime} \mathrm{S}, 80^{\circ} 52^{\prime} \mathrm{W}\right)$. Coll 1977 and subm by K E Stothert, Paleo-Indian Proj, Central Bank of Ecuador, 723 E Woodlawn, San Antonio, Texas.

Tx-3313. OGSE-80 \#1

Shell, Quad G-H/8-9, 90 to $95 \mathrm{~cm}$ level.

$$
7440 \pm 100
$$

Tx-3314. OGSE-80 \#2

$$
7150 \pm 70
$$

Shell, Quad G-H/8, 95 to $100 \mathrm{~cm}$ level.

Tx-3315. OGSE-80 \#3

Shell, Quad G-H $/ 8-9,105$ to $110 \mathrm{~cm}$ level.

Tx-3316. OGSE-80 \#4

Shell, Quad G-H/8, $140 \mathrm{~cm}$ level.

Tx-3318. OGSE-80 Moved bones fraction.

Bone from cemetery in midden. Lab Comment: date is on apatite

Tx-3413. OGSE-80, Feature 24

$8250 \pm 120$ fraction.

Bone from cemetery in midden. Lab Comment: date is on apatite 
General Comment (KES): shell dates in fairly good stratigraphic order excepting Tx-3313 and -3314; dates confirm previous dates from midden (L-1042A, $8600 \pm 200$ BP; L-1042F, $7600 \pm 100$ BP; I-10,097, $8810 \pm 395$ BP; Stothert, pers commun). Bone dates confirm burials made while midden accumulating; Tx-3318 probably corresponds to refuse levels that have eroded from upper level of midden.

\section{Yugoslavia}

\section{Stobi mortar, Series I, Yugoslavia}

Samples of mortar $\left(\mathrm{CaCO}_{3}\right)$ from masonry buildings at Stobi, Hellenistic and Roman site at junction of Crna and Vardar Rivers, S of Titov Veles, Macedonia $\left(41^{\circ} 33^{\prime} \mathrm{N}, 21^{\circ} 59^{\prime} \mathrm{E}\right)$. Previous dates from Stobi are in R, 1975, v 17, p 96-98, and 1977, v 19, p 323. Present series represents first work on application of modified technique for dating of mortar, in which aggregate is removed and only first fraction of $\mathrm{CO}_{2}$ gas evolved from mortar is used (for details of technique see Valastro, 1975; Folk and Valastro, 1975; 1976). Tx-1431 and -1940 to -1944 coll 1973 by Davis (except as noted) and represent lst phase of project. These dates have been reported and discussed in sources just cited (some dates are slightly different here because of being rounded to nearest $10 \mathrm{yr}$ ). Tx-2488 to -2494 coll 1974-5 by Davis, reported here for 1st time. Most samples split and parts prepared and counted independently. In such cases, date is average and dates of parts are given in description. "Corrected dates" are corrected for ${ }^{13} \mathrm{C} /{ }^{12} \mathrm{C}$ and dendrochronology (Damon et al, 1974). Subm by J R Wiseman, Dept Classical Studies, Boston Univ, Boston, Massachusetts, and E M Davis; comments by JRW and EMD.

\section{Tx-1431. Stobi Cent Bas, Folk mortar}

$1870 \pm 180$

Small sample for preliminary experiment in mortar dating. Floor 1, narthex of Central Basilica (formerly called Synagogue Basilica). Coll 1971 by R L Folk. Comment: archaeol date of building construction, AD $460 \pm 30(1490 \pm 30 \mathrm{BP})$, but mortar might be as much as a century later. Mortar ${ }^{14} \mathrm{C}$ date much earlier, but this preliminary experiment justified continuing work on mortar dating.

\section{Tx-1944. Stobi, Cent Bas, Mortar 11}

$1600 \pm 60$

Same provenience as Tx-1431, above, $1628 \pm 50,1560 \pm 90$. Comment: corrected date, $1510 \pm 60$, agrees with archaeol date of building construction, $1490 \pm 30 \mathrm{BP}$; suggests mortar does not refer to time after building was abandoned, as had been suspected.

Tx-1941. Stobi, Theater 2X73, Mortar 4 $\quad \delta^{13} C=-9.3 \%$

Theater, foundation of analemma in $\mathrm{E}$ parodos, $1740 \pm 60,1720 \pm$ $60,1730 \pm 60$. Comment: corrected date is $1690 \pm 40$.

\section{Tx-1942. Stobi, Theater 7X73, Mortar 9} $\mathbf{1 7 2 0} \pm \mathbf{5 0}$

Theater, foundation of $S$ wall of 1st radial corridor of cavea, next to 
$\mathrm{W}$ parodos, $1738 \pm 80,1690 \pm 70,1715 \pm 70$. Comment: corrected date is $1700 \pm 50$.

\section{Tx-2488. Stobi, Theater 1X74}

$1950 \pm 60$

Theater, E Porch II, from core of porch of scene building. Comment: corrected date is $1850 \pm 75$.

\section{Tx-2489. Stobi, Theater $4 \times 74$}

$\mathbf{1 7 8 0} \pm \mathbf{5 0}$ circular corridor. Comment: corrected date is $1775 \pm 70$.

General Comment on $T x-1941,-1942,-2488,-2489$ : samples are all from same construction episode in early 2nd century AD, ca 1840 BP. Average of 4 corrected dates is $1755 \pm 30$. Tx-1941, -1942 agree with each other but are more recent than archaeol date. Tx-2488 agrees well with archaeol date. Tx-2489 is intermediate.

\section{Tx-1943. Stobi, E Bas, Mortar 10}

$$
1660 \pm 50
$$

$\delta^{13} C=-19.2 \%$

Episcopal Basilica, foundation of $S$ wall, $1680 \pm 130,1627 \pm 70$, $1670 \pm 60,1640 \pm 70$. Comment: corrected date is $1550 \pm 50$

\section{Tx-2490. Stobi, E Bas 45X75}

$1600 \pm 70$

Episcopal Basilica, foun corrected date is $1525 \pm 85$.

\section{Tx-2491. Stobi, E Bas 46X75}

$$
1800 \pm 80
$$

$\delta^{13} \mathrm{C}=-18.3 \%$

Episcopal Basilica, foundation of $\mathrm{N}$ wall near $\mathrm{E}$ end. Comment: corrected date is $1625 \pm 85$.

General Comment on Tx-1943, -2490, -2491: samples are all from construction Phase I, 2nd quarter or middle of 5th century AD, ca $1510 \pm$ 20 вP. Average of 3 corrected dates is $1570 \pm 40$. Dates agree with one another within $l \sigma$, and $\mathrm{Tx}-1942$ and -2490 agree with archaeol date; Tx-2491 is early. (Wall 15 ) of Casa Royer of mortar in deposits against exterior of $\mathrm{E}$ wall (Wall 15) of Casa Romana, alt 131.20 to $131.44 \mathrm{~m}$; later than wall, 2015 $\pm 80,2051 \pm 60$. Coll 1972 by J F Cherry. Comment: corrected date is $1880 \pm 60$, much earlier than archaeol date of 4th century aD based on pottery in strata above and below mortar layer; secondary deposition suspected. See general comment, below.

\section{Tx-2492. Stobi, CWE 48X75}

$$
1970 \pm 80
$$

Casa Romana, E (older) part of $S$ wall (Wall 6). Comment: correted date is $1915 \pm 80$. 


\section{Tx-2494. Stobi, CWE 50X75}

Casa Romana, W (younger) part of $\mathrm{S}$ wall (Wall 6). Comment: corrected date is $1865 \pm 85$.

General Comment on Tx-1940, -2492, -2494: age of Casa Romana not known from direct archaeol evidence. Tx-2492 and -2494 indicate 1st century AD, which is compatible with stratigraphic evidence and style of wall decoration. Structural evidence shows that Tx-2492 is earlier than -2494; dates suggest same relationship. Tx-1940, stratigraphically much later, nevertheless agrees with other 2 dates; very likely mortar of Tx-1940 originally came from Casa Romana and was redeposited. Field evidence suggested this possibility.

General Comment on Stobi mortar dates (SV, Jr \& EMD): mortar dates support archaeol dates of times of construction, except in case of Theater where mortar dates vary.

\section{REFERENCES}

Beetham, N and Niering, W, 1961, A pollen diagram from southeastern Connecticut: Am Jour Sci, v 259, p 69-75.

Brain, J P, 1969, Winterville: A case study of prehistoric culture contact in the lower Mississippi Valley: PhD dissert Yale Univ, New Haven, Conn.

Brauner, D R, 1976, Alpowai: the culture history of the Alpowa locality: PhD dissert, Washington State Univ, Pullman.

Damon, P E, Ferguson, C W, Long, A, and Wallick, E I, 1974, Dendrochronologic calibration of the radiocarbon time scale: Am Antiquity, v 39, p 350-366.

Flint, R F and Deevey, E S, 1951, Radiocarbon dating of late Pleistocene events: Am Jour Sci, v 259, p 257-300.

Folk, R L and Valastro, S, Jr, 1975, Radiocarbon dating of mortars at Stobi, in Wiseman, J, ed, Studies in the antiquities of Stobi: Beograd, v 2, p 29-4I.

1976, Successful technique for dating of lime mortar by carbon-14: Jour Field Archaeol, v 3, p 203-208.

Fredlund, L B, 1979, Benson's Butte 24BH1726: Cultural Resources Div, Montana Tech Alumni Foundation, Min Research Center, Repts Investigations no. 8.

Fryxell, R, 1965, Mazama and Glacier Peak volcanic ash layers: relative ages: Science, v 147, p 1288-1290.

Hally, D J, 1966, Post Coles Creek cultural development in the upper Tensas Basin in Louisiana: Southeastern Archaeol Conf Bull, v 6, p 36-40.

Hammett, H H, 1976, Late Quaternary stratigraphy and archaeological chronology in the lower Granite Reservoir, Lower Snake River, Washington: PhD dissert, Washington State Univ, Pullman.

Hansen, H P, 1939, Pollen analysis of a bog in northern Idaho: Am Jour Botany, v 26, p 225-228.

Leonhardy, F C and Rice, D, 1970, A proposed cultural typology for the lower Snake River region, southwestern Washington: Northwest Anthropol Research Notes, Pullman, Washington, v 4, no. 1, p 1-29.

Liddicoat, J C and Coe, R S, 1974, Geomagnetic secular variation recorded in late Pleistocene Lake Lahontan deposits: Am Geophys Union Trans, v 54, p 1109.

Liddicoat, J C, Coe, R S, Lambert, P W, and Valastro, S, Jr, 1979, Paleomagnetic record in late Pleistocene and Holocene dry lake deposits at Tlapacoya, Mexico: Royal Astron Soc Geophys Jour, v 59, p 367-378.

Mack, R N, Okazaki, R, and Valastro, S, Jr, 1979, Bracketing dates for two ash falls from Mount Mazama: Nature, v 279, no. 5710, p 228-229.

Mack, R N, Rutter, N W, Bryant, V M, Jr, and Valastro, S, Jr, 1978a, Re-examination of postglacial vegetational history in northern Idaho: Hager Pond, Bonner Co: Quaternary Research, v 10, no. 2, p 241-255.

Quate, Late Quaternary pollen record from Big Meadow, Pend Oreille County, Washington: Ecology, v 59, no. 5, p 956-966. 
Mack, R N, Rutter, N W, and Valastro, S, Jr, 1978, Late Quaternary pollen record from the Sanpoil River Valley, Washington: Canadian Jour Botany, v 56, no. 14,
p 1642-1650.

1979, Holocene vegetation history of the Okanogan Valley, Washington:
Quaternary Research, v 12, p 212-225.

vegetation history W, Valastro, S, Jr and Bryant, V M, Jr, 1978b, Late Quaternary Gazette, v 139, no. 4, p 499-506.

Mehringer, $\mathbf{P}$ J Science, v 198, p 257-261. 195, P, 1970, Archaeological survey in the lower Yazoo Basin, Mississippi- 1949 1955: Peabody Mus Archaeol Ethnology, Papers, Harvard Univ, v 60.

Rice, D G, 1972, The Windust Phase in lower Snake River prehistory: Lab Anthropol, Washington State Univ, Pullman, Rept Investigation no. 50

Rolingson, M A, 1976, The Bartholomew Phase: A Plaquemine adaptation in the Mississippi Valley, in Cleland, C, ed, Cultural changes and continuity: Essays in honor of James Bennett Griffin: New York, Academic Press.

Rumley, J H, 1956, Plant ecology of a bog in northern Idaho: PhD dissert, Washington State Univ, Pullman

Sackett, M H, 1977, Phytosociology and geological development of three abandoned meanders of the Connecticut River in western Massachusetts: PhD dissert, Dept
Botany, Univ Massachusetts, Amherst.

Schubert, C, Valastro, S, Jr, and Cowart, J B, 1977, Evidencias de levantamiento reciente de la costa norte-central (Cordillera de la Costa), Venezuela: Acta Científicas Venezolana, v 28, no. 6, p 363-372.

Story, D A and Valastro, S, Jr, 1977, Radiocarbon dating and the George C Davis site, Texas: Jour Field Archaeol, v 4, p 63-89.

Thompson, $M$ and Beckett, P H, 1979, A study of prehistoric camp sites in northeast El Paso: New Mexico State Univ, Las Cruces, Rept Texas Hist Comm, Texas
Antiquities Permit 184.

Valastro, S, Jr, 1975, A new technique for the radiocarbon dating of mortars: MA thesis, Dept Geol Sci, Univ Texas, Austin. 